\title{
Quantifying Fraction of Total Power Vs Wavelength of Ultra-Nanoscale Plasmonic Biosensor Device using Metal-Insulator-Metal-Metal Stack, Nano wells and Biotin Layer.
}

\author{
Divya Sharma, R.P. Dwivedi
}

\begin{abstract}
An ultra-thin three-dimensional nanostructured biosensor device based on the Plasmonic principle is custom designed and analyzed for the Plasmonic properties. Here the FDTD (Finite Difference Time Domain) method is adopted as mathematical model using MEEP (MIT Electromagnetic Equation Propagation) open-source simulation tool. The four models are investigated and analyzed in the following order for respective Plasmonic properties of fraction of total power with respect to the wavelength for model-I MIMM layers (Metal-Insulator-Metal-Metal) with no nanostructure (Al$\mathrm{Al}_{2} \mathrm{O}_{3}-\mathrm{Cr}-\mathrm{Au}$ ), model-II MIMM layers with no nanostructure (Al$\mathrm{Al}_{2} \mathrm{O}_{3}-\mathrm{Cr}-\mathrm{Au}$ ) and Biotin layer, model-III MIMM layers (Al$\mathrm{Al}_{2} \mathrm{O}_{3}-\mathrm{Cr}-\mathrm{Au}$ ) with $11 \times 11$ Nano well structures and model-IV MIMM layers with Nano well structures and Biotin layer (Al$\mathrm{Al}_{2} \mathrm{O}_{3}$-Cr-Au-Biotin). Here the structural and functional behavior of model I Vs Model II Vs Model III vs Model IV is simulated and the fraction of power is measured across the biosensor stack layer of MIMM for the wave length range quantified. In model II there is an approximate 5\% power loss at all layers when compared to model I due to addition of the Biotin layer. In model IV there is an approximate $50 \%$ power loss when compared to model III at Au layer, $60 \%$ power loss when compared to model III at Al layer and $67 \%$ of power loss at $\mathrm{Cr}+\mathrm{Al}_{2} \mathrm{O}_{3}$ due to Biotin layer. These quantifications can be used to understand the model and the behavior of the biosensor under various conditions well before the fabrication, thereby reducing the cost and to comprehend the behavior of each material in terms of power dissipation so different material can be experimented.
\end{abstract}

Index Terms: Biosensors, Nanotechnology, Plasmonic, MEEP

\section{INTRODUCTION}

The latest trends in the field of nanotechnology are applied in the field of medicine specifically in the immunodiagnostics [1], [2]. The immunodiagnostics is supported by various types of nanoscale biosensor devices which are currently in the market and also by the new frontiers laid down in the area of biosensor devices that are researched upon constantly [3],[4].

Revised Manuscript Received on 30 July 2019

* Correspondence Author

Divya Sharma, School of Electrical and Computer Science Engineering, Shoolini University, Solan, 173229, H.P., INDIA.

R.P. Dwivedi, School of Electrical and Computer Science Engineering, Shoolini University, Solan, 173229, H.P., INDIA.

(C) The Authors. Published by Blue Eyes Intelligence Engineering and Sciences Publication (BEIESP). This is an open access article under the CC-BY-NC-ND license http://creativecommons.org/licenses/by-nc-nd/4.0/
The carried-out research work is focused on the two areas, label free and labelled biosensors. The latter is used extensively but the processes of diagnostics requires very expensive apparatus

which are not portable, cost of maintenance is very high and laborious because of the need of time bound reagents and chemical compounds stored under specific storage conditions to conduct the diagnostics test [5], [6]. On the other hand, the label free nanoscale biosensor devices based on Plasmonic principle ensures that it would result into portability and cost of maintenance is less expensive and uses minimal reagents and chemical compound [6]. These nanoscale biosensor devices can be reused by performing the standard prescribed clean procedure. The Plasmonic biosensors devices are highly preferred and adopted to different kinds of immunodiagnostics like breast cancer detection, liver cancer detection, etc....for their flexibility, agility and robustness which makes them to acquire grounds on the new arena of medical diagnostics research [7]-[9]. The design and development of nanoscale Plasmonic biosensor has many challenges, two of the key challenges are complex structural and functional design for better optimization [10]-[13]. Since the design, development and fabrication of these Plasmonic sensors require very expensive software for designing and fabrication in sophisticated fabrication labs [14]. It would be good to find alternative low-cost method/approach to keep the research and development cost to minimal, so that the end design of biosensor would be having low cost and would be affordable. To achieve the low cost of development, the open source simulation tool MEEP (MIT Equation Propagation) is used to design and test variety of biosensor designs for structural and functional measurement before going for actual fabrication of the device [15].

\section{METHODOLOGY}

The following section describes the complete methodology under taken, the design of three-dimensional nanoscale biosensor device using simulation tool MEEP (MIT Electromagnetic Equation Propagation, Open Source development tool) [16]-[17]. Using this tool, different Plasmonic based stack layers of different materials of metals, insulators and meta-materials can be designed to target the various immunodiagnostic applications [18]-[22]. 
Here the four different Plasmonic models Model-I, Model-II, Model-III and Model-IV of nanoscale Plasmonic biosensors are discussed in detail, the model-I has Metal-Insulator-Metal-Metal design (MIMM) layer structure with no nanowells/nanoholes. The model-II has Metal-Insulator-Metal-Metal design (MIMM) layer structure with no nanowells and Biotin layer on top. The model-III has Metal-Insulator-Metal-Metal design (MIMM) layer structure with 11x11 nanowells. The model-IV has Metal-Insulator-Metal-Metal design (MIMM) layer structure with 11 x 11 nanowells with Biotin layer. These models I, II, III and IV are investigated for fraction of the power vs wavelength. The detailed design of model-I, model-II, model-III and model-IV are described in the following section,

\section{A. Model -I: Structure design specification}

It is a MIMM structure as shown in figure 1(a) with materials Aluminum, Aluminum oxide, Chromium and Gold layer with the dimension of $25 \mathrm{~nm}, 100 \mathrm{~nm}, 5 \mathrm{~nm}$ and $25 \mathrm{~nm}$ respectively and with the total height of size $155 \mathrm{~nm}$. The figure $1(\mathrm{~b})$ describes the top view with length and breadth of 8.44um and 8.44um respectively.

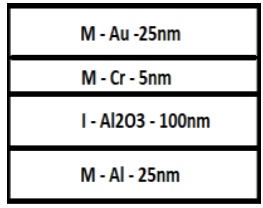

(a)

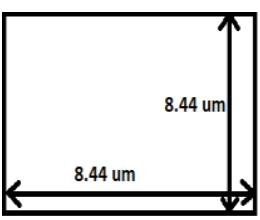

(b)
Fig. 1. Model I Biosensor block design (a) Side view (b) Top view without nanowells structure

\section{B. Model -II: Structure design specification}

It is a MIMM Biotin structure as shown in figure 2(a) with materials Aluminum, Aluminum oxide, Chromium and Gold layer with the dimension of $25 \mathrm{~nm}, 100 \mathrm{~nm}, 5 \mathrm{~nm}, 25 \mathrm{~nm}$ and $50 \mathrm{~nm}$ of Biotin respectively and with the total height of size 205nm. The figure 2(b) describes the top view with length and breadth of 8.44um and 8.44um respectively.

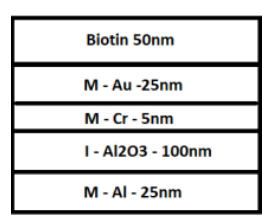

(a)

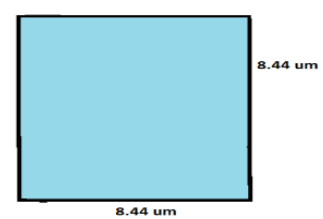

(b)
Fig. 2. Model II Biosensor block design (a) Side view (b) Top view without nanowells structure and Biotin layer

\section{C.Model -III: Structure design specification}

Structure design specification, it is a MIMM structure as shown in figure 3 (a) with materials Aluminum, Aluminum oxide, Chromium and Gold layer with the dimension of 25 $\mathrm{nm}, 100 \mathrm{~nm}, 5 \mathrm{~nm}$ and $25 \mathrm{~nm}$ respectively and with the total height of size $205 \mathrm{~nm}$. It has 11 X 11 nanowells structure with the dimension radius $\mathrm{r}=155 \mathrm{~nm}$, intercell distance $\mathrm{ICD}=340 \mathrm{~nm}$ and well depth of 30nm. The figure 3(b) describes the top view with length and breadth of 8.44um and 8.44um respectively.

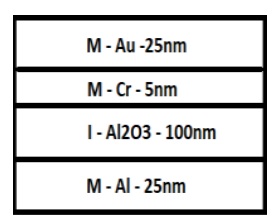

(a)

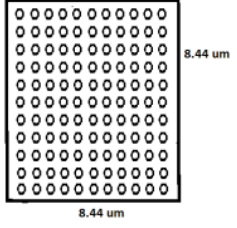

(b)
Fig.3. Model III Biosensor block design (a) Side view (b) Top view with nanowells structure

\section{Model-IV: Structure design specification}

Structure design specification, it is a MIMM structure as shown in figure 4(a) with materials Aluminum, Aluminum oxide, Chromium and Gold layer with the dimension of 25 $\mathrm{nm}, 100 \mathrm{~nm}, 5 \mathrm{~nm}$ and $25 \mathrm{~nm}$ respectively and with the total height of size $155 \mathrm{~nm}$. It has 11 X 11 nanowells structure with the radius of $r=155 \mathrm{~nm}$, intercell distance $\mathrm{ICD}=340 \mathrm{~nm}$ and well depth of 30nm. The Biotin layer of approximately 50nm is coated with the refractive index, epsilon of 2.63. The figure 4(b) describes the top view with length and breadth of 8.44um and 8.44um respectively.

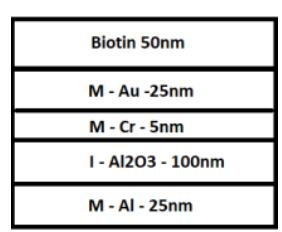

(a)

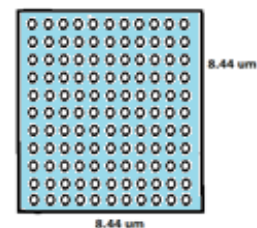

(b)
Fig.4. Model IV Biosensor block design (a) Side view with Biotin layer (b) Top view with nanowells structure and Biotin layer

\section{E. Functional behavior}

The functional behavior of the model I, model II, model III and model IV are simulated and analyzed for the fraction of total power with respect to the wavelength quantified. The source functional parameters include frequency as Gaussian-source, frequency width of $1.25 \times 10^{-13}$, source frequency center of $1.875 \times 10^{-3}$ and source component of Ez. The perfectly matched layer (PML) is added for absorbing the boundary conditions with 800nm width. The flux panels are setup to measure and quantify fraction of total power using the FDTD mathematical model.

\section{MATHEMATICAL MODEL}

There are various mathematical models like Discrete dipole approximation (DDA), FDTD, beam propagation method, etc. that are currently used to understand the behavior of electromagnetics wave propagation and interaction with the materials. Here the MEEP software uses the FDTD model as the core mathematical model of simulation to understand the Model I, II, III and IV for Plasmonic behavior. The finite differential time domain methodology discretizes the time-dependent Maxwell's equations [26], [27]. The electrical field vector and the magnetic field vector components are solved for a given discretize time and space where the partial derivatives are replaced by finite difference approximations using equation (1), (2) and (3). 


$$
\begin{aligned}
& \boldsymbol{\nabla} \times \overline{\mathrm{E}}=-\mu(\delta / \delta \mathrm{t}) \overline{\mathrm{H}} \\
& \boldsymbol{\nabla} \times \overline{\mathrm{H}}=\overline{\mathrm{J}}+\sigma \overline{\mathrm{E}}+\epsilon(\delta / \delta \mathrm{t}) \overline{\mathrm{E}}
\end{aligned}
$$

$\mu(\delta / \delta \mathrm{t}) \mathrm{Hx}=(\delta / \delta \mathrm{z}) \mathrm{Ey}-(\delta / \delta \mathrm{y}) \mathrm{Ez} \quad \epsilon(\delta / \delta \mathrm{t}) \mathrm{Ex}=(\delta / \delta \mathrm{y}) \mathrm{Hz}-(\delta / \delta \mathrm{z}) \mathrm{Hy}-\sigma \mathrm{Ex}$ $\mu(\delta / \delta \mathrm{t}) \mathrm{Hy}=(\delta / \delta \mathrm{x}) \mathrm{Ez}-(\delta / \delta \mathrm{z}) \mathrm{Ex} \quad \epsilon(\delta / \delta \mathrm{t}) \mathrm{Ey}=(\delta / \delta \mathrm{z}) \mathrm{Hx}-(\delta / \delta \mathrm{x}) \mathrm{Hz}-\sigma \mathrm{Ey}$ $\mu(\delta / \delta \mathrm{t}) \mathrm{Hz}=(\delta / \delta \mathrm{y}) \mathrm{Ex}-(\delta / \delta \mathrm{x}) \mathrm{Ey} \quad \epsilon(\delta / \delta \mathrm{t}) \mathrm{Ez}=(\delta / \delta \mathrm{x}) \mathrm{Hy}-(\delta / \delta \mathrm{y}) \mathrm{Hx}-\sigma \mathrm{Ez}$

\section{SIMULATION RESULTS AND DISCUSSION}

The structural and functional simulation are performed using the MEEP simulation tool for the model-I, II, III and IV. The MEEP outputs stores the structural and functional simulation results as part of scientific file format h5 filesystem. The Mayavi 3D visualization tool is used to visualize the simulated results stored in vtk file format, by the conversion of h5 to vtk using the open source h-5util packages [23]-[25]. The figure 5(a) shows the top view simulation of Model I, biosensor block and figure 5(b) show the side view of the same ( $\left.\mathrm{Al}-\mathrm{Al}_{2} \mathrm{O}_{3}-\mathrm{Cr}-\mathrm{Au}\right)$. The $6(\mathrm{a})$ shows the top view simulation of Model II, with Biotin layer on top and figure 6(b) show the side view of the same (Al$\mathrm{Al}_{2} \mathrm{O}_{3}-\mathrm{Cr}-\mathrm{Au}$-Biotin). The figure 7(a) shows the top view simulation of Model III, biosensor MIMM structure block with $11 \times 11$ nano wells and 7(b) show the side view of the same. The figure 8(a) shows the top view simulation of Model $\mathrm{IV}$, with the additional Biotin layer on top (Al$\mathrm{Al}_{2} \mathrm{O}_{3}$-Cr-Au-Biotin) and figure 8 (b) show the side view of the same.

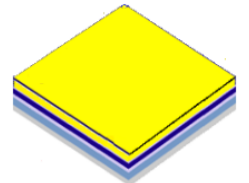

(a)

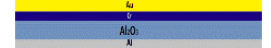

(b)
Fig. 5. Model I Biosensor MIMM structure block simulation (a) top view (b) side view

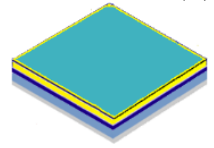

(a)

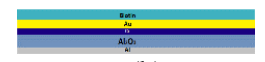

(b)
Fig. 6. Model II Biosensor MIMM structure block with Biotin layer simulation (a) top view (b) side view

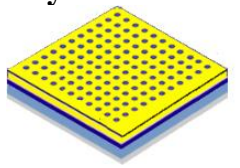

(a)

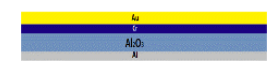

(b)
Fig. 7. Model III Biosensor MIMM structure block with Nano wells simulation (a) top view (b) side view

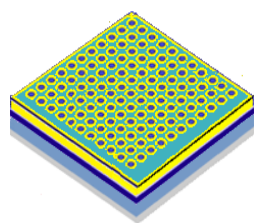

(a) (b)
Fig. 8. Model IV Biosensor MIMM structure block with nanowells and Biotin layer simulation (a) top view (b) side view

The figure 9 shows the biosensor MIMM Model I, functional behavior. The $\mathrm{Al}$ and Au shows the positive fraction of power up to 0.0000015 in the range of the 1.2 to 1.8 um wavelength, but $\mathrm{Cr}$ and $\mathrm{Al}_{2} \mathrm{O}_{3}$ shows the negative shift in power up to 0.0000025 in the same range of 1.2 to $1.8 \mathrm{um}$ wavelength. The figure 10 shows the biosensor MIMM-Biotin Model II, functional behavior. The $\mathrm{Al}$ and $\mathrm{Au}$ shows the positive fraction of power up to 0.000004 in the range of the 1.2 to 1.8 um wavelength, but $\mathrm{Cr}$ and $\mathrm{Al}_{2} \mathrm{O}_{3}$ shows the negative shift in power up to 0.000005 in the same range of 1.2 to $1.8 \mathrm{um}$ wavelength. The figure 11 shows the biosensor MIMM Model III, block functional behavior. The $\mathrm{Al}$ and $\mathrm{Au}$ shows the positive fraction of power up to 0.000003 in the range of the 1.2 to 1.6 um wavelength, but $\mathrm{Cr}$ and $\mathrm{Al}_{2} \mathrm{O}_{3}$ shows the negative shift in power up to 0.000003 in the same range of 1.2 to 1.6 um wavelength. Similarly, the figure 12 shows the biosensor MIMM Model IV, block functional behavior with Biotin layer, the $\mathrm{Al}$ and $\mathrm{Au}$ shows the positive fraction of power up to 0.000004 in the range of the 1.2 to $1.6 \mathrm{um}$ wavelength, but $\mathrm{Cr}$ and $\mathrm{Al}_{2} \mathrm{O}_{3}$ shows the negative shift in power up to 0.000005 in the same range of 1.2 to $1.6 \mathrm{um}$.

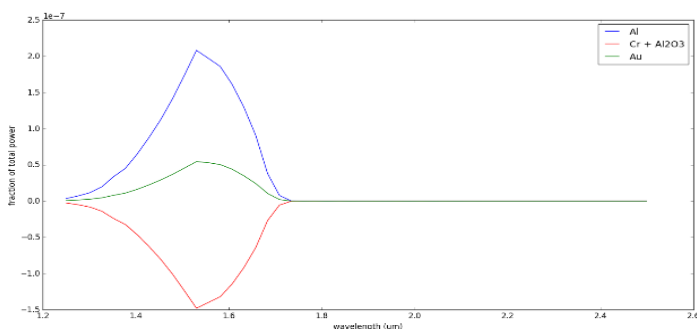

Fig. 9: Model - I, Fraction of total power Vs wavelength without nano wells

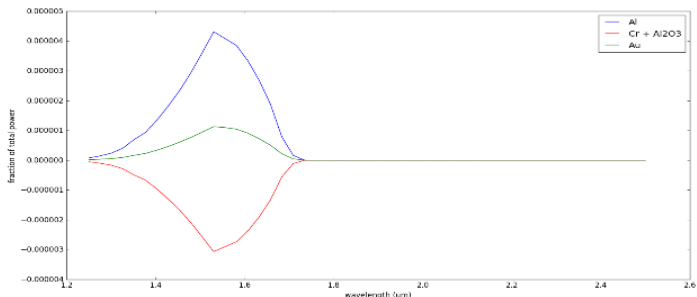

Fig. 10: Model - II, Fraction of total power Vs wavelength without nano wells and with Biotin layer

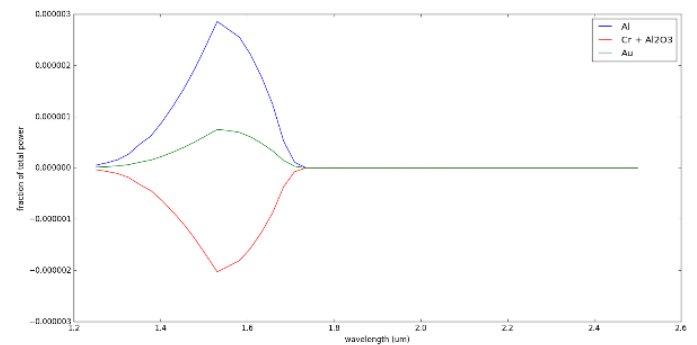

Fig. 11: Model - III, Fraction of total power Vs wavelength with 11 x 11 nanowells 


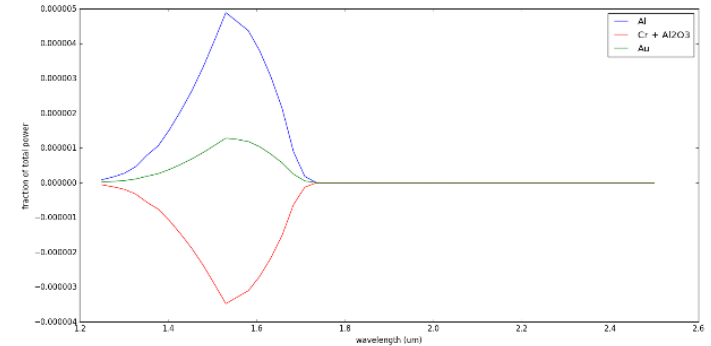

Fig. 12: Model - IV Fraction of total power Vs wavelength with 11 x 11 nanowells and Biotin layer

The table 1 gives the fraction of total power at each layer. In model II there is an approximate 5\% power loss at all layers when compared to Model I due the addition of Biotin layer. Similarly, table 2 shows fraction of power loss between the model III and model IV. There is an approximate $~ 50 \%$ power loss when compared to Model III at Au layer, 60\% power loss when compared to Model III at Al layer and fraction power loss of $67 \%$ difference at $\mathrm{Cr}+\mathrm{Al}_{2} \mathrm{O}_{3}$ due the addition of Biotin layer. Losses can be attributed to surface plasmon polaritons (SPP).

Table - I Model I and Model II fraction of total power percentage comparison

\begin{tabular}{|c|c|c|c|}
\hline Layer & Model I & Model II & $\begin{array}{c}\text { Percentage power } \\
\text { loss because of } \\
\text { Biotin layer in model } \\
\text { II at each layer }\end{array}$ \\
\hline $\mathrm{Au}$ & $5.00 \mathrm{E}-08$ & $1.00 \mathrm{E}-06$ & $\sim 5 \%$ \\
\hline $\mathrm{Al}$ & $2.00 \mathrm{E}-07$ & $4.00 \mathrm{E}-06$ & $\sim 5 \%$ \\
\hline $\begin{array}{c}\mathrm{Cr}+ \\
\mathrm{Al}_{2} \mathrm{O}_{3}\end{array}$ & $-1.50 \mathrm{E}-07$ & $-3.00 \mathrm{E}-06$ & $\sim 5 \%$ \\
\hline
\end{tabular}

Table - 2 Model III and Model IV fraction of total power percentage comparison

\begin{tabular}{|c|c|c|c|}
\hline Layer & Model III & Model IV & $\begin{array}{c}\text { Percentage power loss } \\
\text { because of Biotin } \\
\text { layer in model IV at } \\
\text { each layer }\end{array}$ \\
\hline $\mathrm{Au}$ & $5.00 \mathrm{E}-07$ & $1.00 \mathrm{E}-06$ & $\sim 50 \%$ \\
\hline $\mathrm{Al}$ & $3.00 \mathrm{E}-06$ & $5.00 \mathrm{E}-06$ & $\sim 60 \%$ \\
\hline $\begin{array}{c}\mathrm{Cr}+ \\
\mathrm{Al}_{2} \mathrm{O}_{3}\end{array}$ & $-2.00 \mathrm{E}-06$ & $-3.00 \mathrm{E}-06$ & $\sim 67 \%$ \\
\hline
\end{tabular}

\section{CONCLUSION}

The Nanoscale biosensor design and development is a very complex approach and also expensive, by using the opensource free MEEP tool for design and modelling the biosensor device would help in drastic cut down of the development cost. Here the structural and functional behavior of model I Vs Model II Vs Model III vs Model IV is simulated and the fraction of power is measured across the biosensor stack layer of MIMM for the wave length range quantified. In model II there is an approximate 5\% power loss at all layers when compared to model I due to addition of the Biotin layer. In model IV there is an approximate $50 \%$ power loss when compared to model III at Au layer, 60\% power loss when compared to model III at $\mathrm{Al}$ layer and $67 \%$ of power loss at $\mathrm{Cr}$
$+\mathrm{Al}_{2} \mathrm{O}_{3}$ due to Biotin layer. These quantifications can be used to understand the model and understand the behavior of the biosensor under various conditions well before the fabrication. By quantifying the power loss at individual layer, we can easily understand the behavior of each material in terms of power dissipation, there by different material can be experimented to understand and choose optimal material type. This can be extended to understand the impact of various nanoscale patterns like pyramids, hexagonal nanoholes or nanopillars etc. and their associated power loss for better design of the biosensor device. The novelty in this paper is that the same methodology can be applied to investigate the broad class of other Plasmonic devices with different materials stack, structure and design with different Nano structures to address not just the need of class of immuno-diagnostics applications but also class of Plasmonic nanoscale devices that are used in the nonmedical areas like computing devices power requirement.

\section{REFERENCES}

1. S. Vashist, "Point-of-Care Diagnostics: Recent Advances and Trends", Biosensors, vol. 7, no. 4, p. 62, 2017. Available: 10.3390/bios7040062

2. S. Song, H. Xu and C. Fan, "Potential diagnostic applications of biosensors: current and future directions", International Journal of Nanomedicine, vol. 1, no. 4, pp. 433-440, 2006. Available: 10.2147/nano.2006.1.4.433.

3. S. Chan, Y. Li, L. Rothberg, B. Miller and P. Fauchet, "Nanoscale silicon microcavities for biosensing", Materials Science and Engineering: C, vol. 15, no. 1-2, pp. 277-282, 2001. Available: 10.1016/s0928-4931(01)00219-3.

4. D. Dey and T. Goswami, "Optical Biosensors: A Revolution Towards Quantum Nanoscale Electronics Device Fabrication", Journal of Biomedicine and Biotechnology, vol. 2011, pp. 1-7, 2011. Available: 10.1155/2011/348218

5. R. Higgs, M. Knierman, V. Gelfanova, J. Butler and J. Hale, "Comprehensive Label-Free Method for the Relative Quantification of Proteins from Biological Samples", Journal of Proteome Research, vol. 4, no. 4, pp. 1442-1450, 2005. Available: 10.1021/pr050109b.

6. A. Syahir, K. Usui, K. Tomizaki, K. Kajikawa and H. Mihara, "Label and Label-Free Detection Techniques for Protein Microarrays", Microarrays, vol. 4, no. 2, pp. 228-244, 2015. Available: 10.3390/microarrays4020228.

7. Y. Yao, B. Yi, J. Xiao and Z. Li, "Surface Plasmon Resonance Biosensors and its Application", 2007 1st International Conference on Bioinformatics and Biomedical Engineering, 2007. Available: 10.1109/icbbe.2007.270.

8. Y. Liu et al., "Theoretical Design of Plasmonic Refractive Index Sensor Based on the Fixed Band Detection", IEEE Journal of Selected Topics in Quantum Electronics, vol. 25, no. 2, pp. 1-6, 2019. Available: 10.1109/jstqe.2018.2827661.

9. N. Chiu, T. Wey, C. Yu, T. Hsiao and C. Lin, "Advanced Plasmonic Biosensing Devices and Automation Systems for Disease Diagnostic and Drug Screening Applications", 2008 Eighth International Conference on Intelligent Systems Design and Applications, 2008. Available: 10.1109/isda.2008.39.

10. Wenhua Gu, Li Peng, Senfeng Lai and Yanghui Wu, "The application of surface plasmon polariton effect in photonic integration", 2015 14th International Conference on Optical Communications and Networks (ICOCN), 2015. Available: 10.1109/icocn.2015.7203753.

11. J. Chen et al., "Tunable Resonances in the Plasmonic Split-Ring Resonator", IEEE Photonics Journal, vol. 6, no. 3, pp. 1-6, 2014. Available: 10.1109/jphot.2014.2323294.

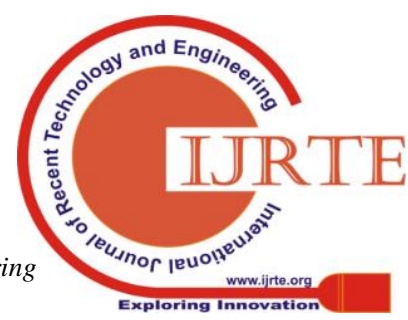


12. Z. Jakšić, "Nanomembrane-based plasmonics", Journal of Nanophotonics, vol. 5, no. 1, p. 051818, 2011. Available: 10.1117/1.3609273.

13. Xiao-Ping Jin, Xu-Guang Huang, Jin Tao, Xian-Shi Lin and Qin Zhang, "A Novel Nanometeric Plasmonic Refractive Index Sensor", IEEE Transactions on Nanotechnology, vol. 9, no. 2, pp. 134-137, 2010. Available: 10.1109/tnano.2009.2038909.

14. H. Pereira, J. Rocha, L. Caballero and O. Neto, "Photonic crystal design tool", 2017 32nd Symposium on Microelectronics Technology and Devices (SBMicro), 2017. Available: 10.1109/sbmicro.2017.8113020.

15. A. Oskooi, D. Roundy, M. Ibanescu, P. Bermel, J. Joannopoulos and S. Johnson, "Meep: A flexible free-software package for electromagnetic simulations by the FDTD method", Computer Physics Communications, vol. 181, no. 3, pp. 687-702, 2010. Available: 10.1016/j.cpc.2009.11.008.

16. P. Sharma, Fleming Dackson Gudagunti and P. Sharan, "An analysis of quality factor for different bio-analytes by using photonic crystal based sensor", 2014 IEEE International Conference on Advanced Communications, Control and Computing Technologies, 2014. Available: 10.1109/icaccct.2014.7019229.

17. K. Rao, Dattathreya and A. Tiwari, "Detection of cancer cell with light wave propagation in optical bio-sensor", 2016 IEEE Conference on Recent Advances in Lightwave Technology (CRALT), 2016. Available: 10.1109/cralt.2016.8066048.

18. J. Zhao, X. Zhang, C. Yonzon, A. Haes and R. Van Duyne, "Localized surface plasmon resonance biosensors", Nanomedicine, vol. 1, no. 2, pp. 219-228, 2006. Available: 10.2217/17435889.1.2.219.

19. P. Sharma and P. Sharan, "An optical sensor for propagation analysis of Lymphocyte cell for cancer cell detection", 2014 IEEE Global Humanitarian Technology Conference - South Asia Satellite (GHTC-SAS), 2014. Available: 10.1109/ghtc-sas.2014.6967565.

20. K. Le, Q. Ngo and T. Nguyen, "Nanostructured Metal-Insulator-Metal Metamaterials for Refractive Index Biosensing Applications: Design, Fabrication, and Characterization", IEEE Journal of Selected Topics in Quantum Electronics, vol. 23, no. 2, pp. 388-393, 2017. Available: 10.1109/jstqe.2016.2615944.

21. J. Fiala, P. Kwiecien and I. Richter, "Physics and design possibilities of plasmonic-based fishnet metamaterial structures", 18th Czech-Polish-Slovak Optical Conference on Wave =

22. P. Sharma, S. Roy and P. Sharan, "Design and simulation of photonic crystal based biosensor for detection of different blood components", 2014 IEEE REGION 10 SYMPOSIUM, 2014. Available: 10.1109/tenconspring.2014.6863019.

23. P. Ramachandran and G. Varoquaux, "Mayavi: 3D Visualization of Scientific Data", Computing in Science \& Engineering, vol. 13, no. 2, pp. 40-51, 2011. Available: 10.1109/mcse.2011.35.

24. Y. Wang, W. Jiang and G. Agrawal, "SciMATE: A Novel MapReduce-Like Framework for Multiple Scientific Data Formats", 2012 12th IEEE/ACM International Symposium on Cluster, Cloud and Grid Computing (ccgrid 2012), 2012. Available: 10.1109/ccgrid.2012.32.

25. M. Folk, R. McGrath and N. Yeager, "HDF: an update and future directions", IEEE 1999 International Geoscience and Remote Sensing Symposium. IGARSS'99 (Cat. No.99CH36293). Available: 10.1109/igarss.1999.773469.

26. K. Yee and J. Chen, "The finite-difference time-domain (FDTD) and the finite-volume time-domain (FVTD) methods in solving Maxwell's equations", IEEE Transactions on Antennas and Propagation, vol. 45, no. 3, pp. 354-363, 1997. Available: 10.1109/8.558651.

27. K. Jung, W. Yoon, Y. Park, P. Berger and F. Teixeira, "Broadband Finite-Difference Time-Domain Modeling of Plasmonic Organic Photovoltaics", ETRI Journal, vol. 36, no. 4, pp. 654-661, 2014 Available: 10.4218/etrij.14.0113.0767 